\title{
Utility of Frozen Section in the Evaluation of Borderline Ovarian Tumors: A Single Institution Experience
}

\author{
Marilyn Huang ${ }^{1, *}$, Matthew Schlumbrecht ${ }^{1}$, Tegan Hunter ${ }^{2}$, Mehrdad Nadji ${ }^{3}$, Andre Pinto ${ }^{3}$ \\ ${ }^{1}$ Department of Obstetrics \& Gynecology, University of Miami, Miami, USA \\ ${ }^{2}$ Miller School of Medicine, University of Miami, Miami, USA \\ ${ }^{3}$ Department of Pathology, University of Miami, Miami, USA
}

Email address:

m.huang@med.miami.edu (M. Huang), mschlumbrecht@miami.edu (M. Schlumbrecht), thunter@med.miami.edu (T. Hunter), mnadji@miami.edu (M. Nadji), apinto@miami.edu (A. Pinto)

${ }^{*}$ Corresponding author

\section{To cite this article:}

Marilyn Huang, Matthew Schlumbrecht, Tegan Hunter, Mehrdad Nadji, Andre Pinto. Utility of Frozen Section in the Evaluation of Borderline Ovarian Tumors: A Single Institution Experience. Journal of Gynecology and Obstetrics. Vol. 7, No. 2, 2019 , pp. 41-45. doi: $10.11648 /$ j.jgo.20190702.13

Received: March 3, 2019; Accepted: April 9, 2019; Published: May 6, 2019

\begin{abstract}
Background: Borderline ovarian tumors (BOTs) account for a $10-15 \%$ subset of all primary ovarian epithelial neoplasms. Preoperative imaging and serologic markers are often inconclusive at distinguishing between benign, premalignant, and malignant ovarian tumor. Limitations at time of frozen section (FS) are relatively well known, and misinterpretation may occur potentially leading to over- and under-treatment. We evaluated all cases of BOTs submitted for FS in our institution to determine the accuracy of intraoperative diagnosis when compared with the final pathology, and possibly identify features that may guide surgical staging decision-making. Methods: We identified all intraoperative diagnoses of BOTs from our institution in a 12-year period. Clinical and pathologic data were abstracted. Intraoperative pathology diagnosis was compared to final pathologic diagnosis. Statistical analysis was performed using chi-square and logistic regression. Results: There were 80 cases included for analyses, of which 39 (48.8\%) were serous borderline tumor (SBT), 18 (22.5\%) mucinous borderline tumors (MBT), $1(1.2 \%)$ endometrioid borderline tumor, and $22(27.5 \%)$ at least borderline tumor (of various histologies). There were 13 cases with a discrepancy between FS and final diagnosis. In patients with a discrepancy where final pathology demonstrated carcinoma, 4/11 (36.3\%) were not staged or had incomplete staging. Subsequently, 3/4 (75\%) underwent a re-operation for staging purposes. In patients with discrepant pathology, discrepancy was more common $8 / 37$ $(21.6 \%)$ among non-gynecologic pathologists compared to 5/43 (11.6\%) among gynecologic pathologists, but not statistically significant $(\mathrm{p}=0.23)$. When "at least borderline" tumor was diagnosed at FS, 10/22 (45\%) had invasive malignancies on final pathology compared to diagnosis of BOT "only" on FS; on which $1 / 58(1.7 \%)$ had invasive carcinoma. The cases with histologic diagnosis of BOT "only" were associated with significantly reduced discrepancy (OR 0.04 [95\% CI 0.01-0.18], p< 0.001). Conclusion: In conclusion, use of intraoperative evaluation for ovarian tumors is a useful diagnostic tool but has its limitations. In intraoperative cases where pathologists call "at least borderline", strong consideration for surgical staging should be contemplated with re-evaluation of preoperative testing. Moreover, when possible, direct communication between surgeon and pathologist at time of FS diagnosis of BOT may be valuable.
\end{abstract}

Keywords: Ovarian Tumor, Borderline, Intraoperative Diagnosis, Frozen Section

\section{Introduction}

Ovarian cancer is the most common lethal gynecologic malignancy in women [1]. Borderline ovarian tumors (BOTs) account for a $10-15 \%$ subset of all primary ovarian epithelial neoplasms [2]. BOTs typically occur in women at younger ages, with about $25 \%$ diagnosed younger than 35 years, and at earlier stages $[3,4]$. Preoperative imaging and serologic markers are often inconclusive at distinguishing between benign, pre-malignant, and malignant ovarian tumors [5-7]. 
However, precise characterizations of BOTs are imperative in guiding clinical and surgical management, particularly in women desiring fertility $[8,9]$.

Retrospective studies have not demonstrated significant differences in recurrence and survival between staged and unstaged women with BOTs; thus suggesting limited benefit to surgical staging [10-12]. The use of intraoperative evaluation through frozen sections (FS) has been used to determine the necessity of surgical staging in preoperative ovarian tumors. Nevertheless, with frozen section, there is a risk of inaccurate diagnosis leading to understaging women with presumed early stage malignancy (false negative) or overstaging women without malignancy (false positive). Analysis of ways to improve the accuracy and usefulness of frozen section are essential. The objective of this study was to evaluate intraoperative features that may guide surgical decision-making.

\section{Material and Methods}

The surgical pathology electronic database was reviewed from 2003-2015 at our institution, after Institutional Research Board approval. All ovarian neoplasm cases containing a frozen section diagnosis of BOT were included. Clinical variables were abstracted from the institutional electronic medical record system. Data points identified included preoperative CA125 value, age at diagnosis, unilateral or bilateral tumor, and type of surgery performed. Pathologic factors evaluated were diagnosis at frozen section, final (permanent) diagnosis, number of frozen section, size of tumor specimen, presence of extra-ovarian disease, and sub-specialty of pathologist doing frozen section interpretation. Frozen section diagnoses were compared to final pathology results. At time of FS, diagnosis of benign, BOT (with specific histology if able), at least BOT, or carcinoma was provided. The term "at least BOT" was used if the pathologist suspected invasive malignancy but did not have sufficient histologic evidence on FS sampling to definitively distinguish between BOT and carcinoma. Cases with discordant diagnoses (defined as different diagnoses in frozen section versus permanent, including cases classified as "at least borderline" on FS but on which the final pathology showed invasive carcinoma) were re-reviewed by one pathologist (AP). The presence of invasion was defined as stromal infiltration of neoplastic cells spanning $5.0 \mathrm{~mm}$ or more in linear extent or $10.0 \mathrm{~mm}^{2}$ or more in total area, as well as mucinous carcinomas showing an expansile pattern of invasion.

Statistical analyses were performed using STATA IC 14 (StataCorp, College Station, TX). Summary statistics were used to describe the patient cohort. Chi-square testing (or Fisher's exact test when appropriate) was used to analyze proportional associations between categorical variables. The Wilcoxon ran-sum test was used for non-parametric comparisons. To identify factors which may be associated with discrepancy between frozen section evaluation and final pathologic interpretation, logistic regression analyses were performed, and 95\% confidence intervals generated. All tests were two-sided, and p-values $<0.05$ were considered statistically significant.

\section{Results}

There were 80 cases included for analyses, of which 39 (48.8\%) were serous borderline tumor (SBT), 18 (22.5\%) mucinous borderline tumors (MBT), 1 (1.2\%) endometrioid borderline tumor, and $22(27.5 \%)$ at least borderline tumor (of various histologies). The most common final pathologic diagnoses were SBTs (56.3\%) and MBTs (26.2\%). The majority of women $45 / 80(56.3 \%)$ were premenopausal $(<50$ years), with $12 / 80$ (15\%) being less than 30 years. In most cases, greater than 1 frozen section was obtained $44 / 80$ (55\%). Tumor size greater than or equal to $10 \mathrm{~cm}$ occurred in $61.2 \%$ of cases. The majority of cases were operated on by a gynecologic oncologist (85\%). CA125 levels were not obtained in $24(30 \%)$ of cases, but greater than 35 (abnormal) in $37(46.2 \%)$. Staging procedures were performed in the majority of cases $(61 / 80,76.3 \%)$. Tumor characteristics are summarized in Table 1.

Table 1. Characteristics of evaluated tumors $(n=80)$.

\begin{tabular}{|c|c|}
\hline & Number (\%) \\
\hline \multicolumn{2}{|l|}{ Age } \\
\hline$<30$ years & $12(15)$ \\
\hline $30-50$ years & $33(41.3)$ \\
\hline$\geq 51$ years & $35(43.7)$ \\
\hline \multicolumn{2}{|l|}{ Frozen section diagnosis } \\
\hline At least borderline tumor & $22(27.5)$ \\
\hline Serous borderline tumor & $39(48.8)$ \\
\hline Mucinous borderline tumor & $18(22.5)$ \\
\hline Endometrioid borderline tumor & $1(1.2)$ \\
\hline \multicolumn{2}{|l|}{ Final diagnosis } \\
\hline Serous borderline tumor & $45(56.3)$ \\
\hline Mucinous borderline tumor & $21(26.2)$ \\
\hline Low-grade serous carcinoma & $2(2.5)$ \\
\hline Mucinous carcinoma & $3(3.8)$ \\
\hline Endometrioid carcinoma & $1(1.2)$ \\
\hline High-grade serous carcinoma & $4(5)$ \\
\hline Cystadenoma (benign) & $2(2.5)$ \\
\hline Other & $2(2.5)$ \\
\hline \multicolumn{2}{|c|}{ Number of frozen sections obtained } \\
\hline 1 & $36(45)$ \\
\hline$\geq 1$ & $44(55)$ \\
\hline \multicolumn{2}{|l|}{ CA125 } \\
\hline$<35$ & $19(23.8)$ \\
\hline$\geq 35$ & $37(46.2)$ \\
\hline Unknown & $24(30)$ \\
\hline \multicolumn{2}{|l|}{ Tumor size (cm) } \\
\hline$<10$ & $31(38.8)$ \\
\hline $10-20$ & $31(38.8)$ \\
\hline$\geq 20$ & $18(22.4)$ \\
\hline \multicolumn{2}{|l|}{ Laterality } \\
\hline Unilateral & $42(52.5)$ \\
\hline Bilateral & $32(40)$ \\
\hline Unknown & $6(7.5)$ \\
\hline \multicolumn{2}{|l|}{ Extra-ovarian disease } \\
\hline Yes & $22(27.5)$ \\
\hline No & $55(72.5)$ \\
\hline \multicolumn{2}{|l|}{ Debulking/Staging performed } \\
\hline Yes & $61(76.3)$ \\
\hline No & $16(23.7)$ \\
\hline
\end{tabular}


There were 13 cases with a discrepancy between frozen section and final diagnosis. See Figure 1 for discrepancy.
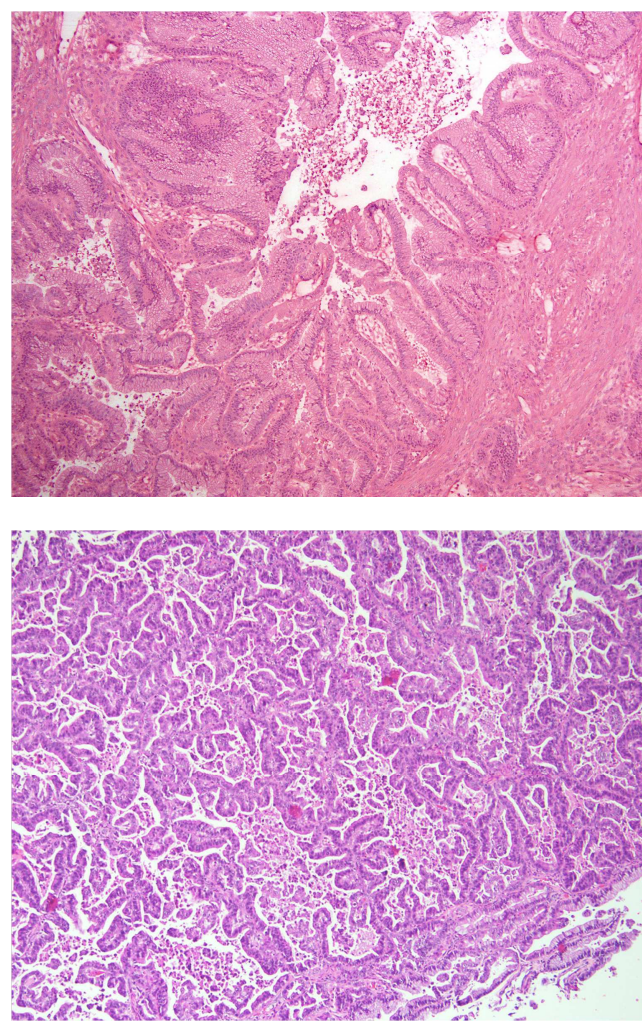

Figure 1. (Top) Frozen section of mucinous tumor initially classified as borderline. Despite some areas of epithelial complexity, it lacked invasion in the sampled tissue (100x magnification). (Bottom) Permanent section from the same case demonstrating invasive mucinous carcinoma with a confluence of the neoplastic glands, diagnostic of expansile pattern of invasion (hematoxylin and eosin, 100x magnification).

'Of the 13 cases, 11 were upstaged on final diagnosis to invasive malignancy and two were downgraded to benign (cystadenoma). In the two cystadenoma cases, one was surgically staged with lymph nodes while the other case was not staged. In patients with a discrepancy where final pathology demonstrated carcinoma, 4/11 (36.3\%) were not staged or had incomplete staging. Subsequently, $3 / 4$ of these patients $(75 \%)$ underwent a re-operation for staging purposes. In women with discrepant pathology, the frozen section was more commonly performed by non-gynecologic pathologists $(8 / 37,21.6 \%)$ when compared to $5 / 43(11.6 \%)$ among gynecologic pathologists, but not statistically significant $(\mathrm{p}=0.23)$. When "at least borderline" tumor was diagnosed at frozen section, 10/22 (45\%) had invasive malignancies on final pathology compared to diagnosis of BOT on frozen section; only $1 / 58(1.7 \%)$ had invasive malignancy. The cases with a histologic diagnosis of borderline tumor "only" were associated with significantly reduced discrepancy on final pathology compared to "at least" BOT diagnosis (OR 0.04 [95\% CI 0.01-0.18], p< 0.001). Furthermore, in the tumors called at least BOT, only 4/22 (18\%) had known CA 125 results, whereas in tumors called "BOT" 20/58 (34\%) had known CA 125 ( $\mathrm{p}=0.16)$. There was no significant difference in the number of frozen sections between discrepant and non-discrepant cases $(\mathrm{p}=0.16)$. Table 2 summarizes predictors of discrepancy between frozen section and final pathology.

Table 2. Predictors of discrepancy between final pathology and frozen section.

\begin{tabular}{|c|c|c|}
\hline Variable & Unadjusted OR $(95 \% \mathrm{CI})$ & p-value \\
\hline Age at diagnosis & $0.99(0.95-1.04)$ & 0.77 \\
\hline \multicolumn{3}{|l|}{ Pathologist interpreting frozen } \\
\hline \multicolumn{3}{|l|}{ Non-GYN pathologist (ref) } \\
\hline GYN pathologist & $0.47(0.14-1.16)$ & 0.23 \\
\hline \multicolumn{3}{|l|}{ Number of frozen sections } \\
\hline \multicolumn{3}{|l|}{ One (ref) } \\
\hline More than one & $2.06(0.58-7.34)$ & 0.27 \\
\hline \multicolumn{3}{|l|}{ CA125 } \\
\hline \multicolumn{3}{|l|}{$<35$ (ref) } \\
\hline$\geq 35$ & $0.83(0.18-3.93)$ & 0.82 \\
\hline \multicolumn{3}{|l|}{ CA125 Knowledge } \\
\hline \multicolumn{3}{|l|}{ Known value (ref) } \\
\hline Unknown value & $1.58(0.45-5.44)$ & 0.47 \\
\hline \multicolumn{3}{|l|}{ Frozen Histology } \\
\hline \multicolumn{3}{|l|}{ At least borderline (ref) } \\
\hline Confirmed borderline & $0.04(0.01-0.18)$ & $<0.001$ \\
\hline \multicolumn{3}{|l|}{ Tumor size } \\
\hline$\geq 10 \mathrm{~cm}$ & $1.51(0.42-5.43)$ & 0.52 \\
\hline \multicolumn{3}{|l|}{ Laterality } \\
\hline \multicolumn{3}{|l|}{ Unilateral (ref) } \\
\hline Bilateral & $0.92(0.31-2.78)$ & 0.89 \\
\hline \multicolumn{3}{|l|}{ Disease localization } \\
\hline No extra-ovarian disease (ref) & & \\
\hline Extra-ovarian disease & $0.83(0.20-3.44)$ & 0.80 \\
\hline
\end{tabular}

There were 39 SBTs diagnosed on frozen section, none of which were upgraded to carcinoma on final pathology. In the 18 frozen sections called mucinous borderline tumors (MBTs), one was upstaged to mucinous adenocarcinoma and one downgraded to mucinous cystadenoma (no significant difference compared to SBT, $\mathrm{p}=0.23$ ). All cases of invasive serous carcinomas were initially called "at least borderline" tumor on frozen section. Both BOTs cases that were downgraded on final pathology corresponded to mucinous lesions (Table 3).

Table 3. Tumors of discrepant histology ( $B T=$ borderline tumor).

\begin{tabular}{lll}
\hline Frozen Section & Final Pathology & $\begin{array}{l}\text { Staging Procedure } \\
\text { Performed* }\end{array}$ \\
\hline Serous BT & $\begin{array}{l}\text { Serous carcinoma, unspecified } \\
\text { grade }\end{array}$ & Yes \\
Mucinous BT & Mucinous carcinoma & No \\
At least BT & Endometrioid carcinoma & Incomplete (no nodes) \\
At least BT & Mucinous carcinoma & Yes \\
At least BT & High-grade serous carcinoma & Incomplete (no nodes) \\
At least BT & Low-grade serous carcinoma & Yes \\
At least BT & High-grade serous carcinoma & Yes \\
At least BT & Low-grade serous carcinoma & No \\
At least BT & Mucinous carcinoma & Yes \\
At least BT & High-grade serous carcinoma & No \\
At least BT & High-grade serous carcinoma & Unknown \\
Mucinous BT & Mucinous cystadenoma & No \\
At least BT & Mucinous cystadenoma & Yes \\
\hline
\end{tabular}

*Staging indicates anything beyond hysterectomy/BSO or ovarian cystectomy. If lymph node dissection indicated per histology but not performed, staging deemed incomplete. 


\section{Discussion}

Frozen section is an important diagnostic intraoperative tool in assessing ovarian masses. Benefits of intraoperative frozen section include decreased surgical morbidity associated with unnecessary surgical staging and reducing the need for second surgical procedure to complete surgical staging $[13,14]$. The accuracy of frozen section in classifying tumors as benign, borderline, or invasive is crucial to providing optimal surgical treatment. Factors that may contribute to a discordant diagnosis at the time of frozen section include lack of sampling in key areas, the number of sections sampled, and the experience of the pathologist.

In our study, we found that while there were more discrepant cases among non-gynecologic pathologists compared to gynecologic pathologists, this was not statistically significant and is similar to current literature. Prior studies have highlighted the diagnostic challenges of borderline tumors on frozen section, leading to the use of qualifying terms in frozen reports such as rule out borderline or at least borderline [15-17]. In a retrospective study evaluating the accuracy of frozen section, sensitivities reported were $99.7 \%, 89.5 \%$, and $96.3 \%$ for benign, borderline, and malignant tumors by gynecologic pathologists compared to $97 \%, 50 \%$, and $84.6 \%$ among non-gynecologic pathologists [13]. Similarly for the same cohort, specificities were $97.6 \%, 85 \%$, and $99 \%$ among gynecologic pathologists versus $95.2 \%, 96.2 \%$, and $94.5 \%$ in non-gynecologic pathologists. The overall accuracy of frozen section rate was $97.1 \%$ with an increased rate if evaluated by gynecologic pathologist [13]. In another study, the predictive values for benign, borderline, and carcinoma on frozen section were $86.1 \%, 66.6 \%$ and $100 \%$ respectively [18]. Additionally, for frozen section with diagnosis of borderline tumor, there was a $33.3 \%$ chance that the final diagnosis would be carcinoma on permanent section. The authors concluded that frozen section should be used cautiously in borderline tumors, particularly in older patients and in mucinous tumors [18]. Other studies suggest that frozen section is insufficient for accurate diagnosis of BOTs due to risk of underdiagnosis $[19,20]$. The present study highlights the continued challenges of borderline tumors diagnosis at FS with 13 discrepant cases, 3 of whom, required additional surgery.

The role of surgical staging in invasive ovarian carcinoma is well established, while surgical staging in BOTs is no longer supported by the literature [21, 22]. This change in practice pattern highlights the importance of accurate intraoperative frozen section to minimize the number of unstaged patients and/or patients requiring a second surgical procedure. In a large database review by Ratnavelu et al, in over 4000 cases, the largest discordance was in reporting BOT at time of frozen section. The authors went on to suggest that investigation into factors leading to discordance and standardization for reporting borderline tumor may improve accuracy [23]. Our data further underscores the limitations of frozen section in BOTs, particularly in mucinous BOTs [24].

In our study, $16.3 \%$ of cases were discrepant, with the majority being underdiagnosed (84.6\%), and 38.5\% involved mucinous tumors. This is consistent with previously reported data $[18,25]$. Furthermore, $3.8 \%$ of patients in our study underwent a second surgical staging procedure, which may be lower than other studies, because the majority of our patients were initially operated on by a gynecologic oncologist. However, when pathologists in our cases called frozen section "at least borderline", $45 \%$ were invasive malignancies on final pathology. In these cases, direct communication between surgeon and pathologist as well as consideration of other preoperative testing in combination with frozen section may guide decision for surgical staging.

Our study is limited by weakness inherent to all retrospective studies. While most other single institution studies report similar number of cases, other larger multiinstitutional or meta-analysis present significantly more patients. Due to the timeframe of the study, which encompassed transitioning to electronic medical records, complete records were not available for all patients (ie CA 125). Strengths of the study include the 12-year time interval and surgeries performed by gynecologic oncologists. Surgeon bias is limited in our study as the majority of surgeons were gynecologic oncologists and able to complete staging procedures. Additionally, all discordant cases were reviewed by a single pathologist to confirm diagnosis.

\section{Conclusion}

In conclusion, use of intraoperative evaluation for ovarian tumors can be a useful diagnostic tool if its limitations are acknowledged. In patients with ovarian tumors, preoperative workup including history, CA125, and imaging, all are critical data points but may not be sufficient in assessing risk of BOT or carcinoma. In intraoperative cases where pathologists call "at least borderline", strong consideration for surgical staging should be contemplated with reevaluation of preoperative testing. Moreover, when possible, direct communication between surgeon and pathologist at time of frozen section diagnosis of BOT may be invaluable in guiding surgical decision making. Additional studies are needed to further explore factors leading to discordant cases, particularly in mucinous BOTs.

\section{References}

[1] American Cancer Society. www.cancer.org, 2016

[2] Zanetta G, Rota S, Chiari S, et al: Behavior of borderline tumors with particular interest to persistence, recurrence, and progression to invasive carcinoma: a prospective study. J Clin Oncol 19:2658-64, 2001

[3] Messalli EM, Grauso F, Balbi G, et al: Borderline ovarian tumors: features and controversial aspects. Eur $\mathrm{J}$ Obstet Gynecol Reprod Biol 167:86-9, 2013

[4] Tinelli R, Tinelli A, Tinelli FG, et al: Conservative surgery for borderline ovarian tumors: a review. Gynecol Oncol 100:18591, 2006. 
[5] Bent CL, Sahdev A, Rockall AG, et al: MRI appearances of borderline ovarian tumours. Clin Radiol 64:430-8, 2009

[6] Medeiros LR, Rosa DD, da Rosa MI, et al: Accuracy of CA 125 in the diagnosis of ovarian tumors: a quantitative systematic review. Eur J Obstet Gynecol Reprod Biol 142:99$105,2009$.

[7] Timmerman D, Van Calster B, Testa AC, et al: Ovarian cancer prediction in adnexal masses using ultrasound-based logistic regression models: a temporal and external validation study by the IOTA group. Ultrasound Obstet Gynecol 36:226-34, 2010.

[8] Lin PS, Gershenson DM, Bevers MW, et al: The current status of surgical staging of ovarian serous borderline tumors. Cancer 85:905-11, 1999.

[9] Tinelli R, Malzoni M, Cosentino F, et al: Feasibility, safety, and efficacy of conservative laparoscopic treatment of borderline ovarian tumors. Fertil Steril 92:736-41, 2009.

[10] Rao GG, Skinner E, Gehrig PA, et al: Surgical staging of ovarian low malignant potential tumors. Obstet Gynecol 104:261-6, 2004.

[11] Trope CG, Kristensen G, Makar A: Surgery for borderline tumor of the ovary. Semin Surg Oncol 19:69-75, 2000.

[12] Winter WE, 3rd, Kucera PR, Rodgers W, et al: Surgical staging in patients with ovarian tumors of low malignant potential. Obstet Gynecol 100:671-6, 2002.

[13] Bige O, Demir A, Saygili U, et al: Frozen section diagnoses of 578 ovarian tumors made by pathologists with and without expertise on gynecologic pathology. Gynecol Oncol 123:43-6, 2011.

[14] Boriboonhirunsarn D, Sermboon A: Accuracy of frozen section in the diagnosis of malignant ovarian tumor. J Obstet Gynaecol Res 30:394-9, 2004.

[15] Menzin AW, Rubin SC, Noumoff JS, et al: The accuracy of a frozen section diagnosis of borderline ovarian malignancy. Gynecol Oncol 59:183-5, 1995.
[16] Robinson WR, Curtin JP, Morrow CP: Operative staging and conservative surgery in the management of low malignant potential ovarian tumors. Int J Gynecol Cancer 2:113-118, 1992.

[17] Twaalfhoven FC, Peters AA, Trimbos JB, et al: The accuracy of frozen section diagnosis of ovarian tumors. Gynecol Oncol 41:189-92, 1991.

[18] Morton R, Anderson L, Carter J, et al: Intraoperative Frozen Section of Ovarian Tumors: A 6-Year Review of Performance and Potential Pitfalls in an Australian Tertiary Referral Center. Int J Gynecol Cancer 27:17-21, 2017.

[19] Bozdag H, Guzin K, Gocmen A, et al: The diagnostic value of frozen section for borderline ovarian tumours. J Obstet Gynaecol 36:626-30, 2016.

[20] Gultekin E, Gultekin OE, Cingillioglu B, et al: The value of frozen section evaluation in the management of borderline ovarian tumors. J Cancer Res Ther 7:416-20, 2011.

[21] Snider DD, Stuart GC, Nation JG, et al: Evaluation of surgical staging in stage I low malignant potential ovarian tumors. Gynecol Oncol 40:129-32, 1991.

[22] Zapardiel I, Rosenberg P, Peiretti M, et al: The role of restaging borderline ovarian tumors: single institution experience and review of the literature. Gynecol Oncol 119:274-7, 2010.

[23] Ratnavelu ND, Brown AP, Mallett S, et al: Intraoperative frozen section analysis for the diagnosis of early stage ovarian cancer in suspicious pelvic masses. Cochrane Database Syst Rev 3:CD010360, 2016.

[24] Matsuo K, Machida H, Mandelbaum RS et al. Mucinous borderline ovarian tumor versus invasive well-differentiated mucinous ovarian cancer: Difference in characteristics \& outcomes. Gynecol Oncol. S0090-8258. 2019.

[25] Shih KK, Zhou Q, Huh J, et al: Risk factors for recurrence of ovarian borderline tumors. Gynecol Oncol 120:480-4, 2011. 